\title{
Dirac Equation under Scalar and Vector Generalized Isotonic Oscillators and Cornell Tensor Interaction
}

\author{
H. Hassanabadi, ${ }^{1}$ E. Maghsoodi, ${ }^{2}$ Akpan N. Ikot, ${ }^{3}$ and S. Zarrinkamar ${ }^{4}$ \\ ${ }^{1}$ Physics Department, Shahrood University, Shahrood 36199-95161-316, Iran \\ ${ }^{2}$ Department of Basic Sciences, Islamic Azad University, Shahrood Branch, Shahrood, Iran \\ ${ }^{3}$ Theoretical Physics Group, Department of Physics, University of Port Harcourt, PMB 5323, Choba, Port Harcourt, Nigeria \\ ${ }^{4}$ Department of Basic Sciences, Islamic Azad University, Garmsar Branch, Garmsar, Iran
}

Correspondence should be addressed to H. Hassanabadi; h.hasanabadi@shahroodut.ac.ir

Received 6 September 2014; Accepted 13 November 2014; Published 30 November 2014

Academic Editor: Shi-Hai Dong

Copyright (c) $2014 \mathrm{H}$. Hassanabadi et al. This is an open access article distributed under the Creative Commons Attribution License, which permits unrestricted use, distribution, and reproduction in any medium, provided the original work is properly cited. The publication of this article was funded by $\mathrm{SCOAP}^{3}$.

Spin and pseudospin symmetries of Dirac equation are solved under scalar and vector generalized isotonic oscillators and Cornell potential as a tensor interaction for arbitrary quantum number via the analytical ansatz approach. The spectrum of the system is numerically reported for typical values of the potential parameters.

\section{Introduction}

The solution of the fundamental dynamical equations is an interesting phenomenon in many fields of physics. The relativistic Dirac equation which describes the motion of spin $1 / 2$ particle [1]. Within the framework of Dirac equation, pseudospin and spin symmetries are used to study features of deformed nuclei, superdeformation, and effective shell model [2]. The concept of SUSYQM provides theoretical physicists with a powerful tool to deal with nonrelativistic Schrödinger equation. The pseudospin symmetry is referred to as a quasidegeneracy of single nucleon doublets with nonrelativistic quantum number $(n, l, j=l+1 / 2)$ and $(n-1, l+2, j=l+3 / 2)$, where $n, l$, and $j$ are single nucleon radial, orbital, and total angular quantum numbers [3] and it was shown that the exact pseudospin symmetry occurs in the Dirac equation when $d \Sigma(r) / d r=0$; that is, $\Sigma(r)=V(r)+S(r)=c_{\mathrm{ps}}=$ const., where $V(r)$ and $S(r)$ are repulsive vector potential and attractive scalar potential, respectively. Details of recent review of spin and pseudospin symmetries are given in [4]. These symmetries, under various phenomenological potentials, have been investigated using various methods such as Nikiforov-Uvarov (NU), supersymmetric quantum mechanics (SUSSYQM) and shape invariance (SI), ansatz approach, and asymptotic iteration (AIM) [5-15]. In recent years, the Dirac equation with different potentials in relativistic quantum mechanics with spin and pseudospin symmetry has been investigated [16-25]. The main aim of the present paper is to obtain approximate solutions of the Dirac equation with scalar and vector generalized isotonic oscillators and Cornell tensor interaction under the above mentioned symmetry limits. The isotonic oscillator potential consists of the harmonic oscillator plus centrifugal barrier which is of great interest in the theory of coherent states [26] and quantum optics [27] and in the analysis of the isochronous oscillator [28]. This potential is important because of its relation with supersymmetric quantum mechanics [29]. The two-dimensional version of the isotonic potential is superintegrable and usually is referred to as the Smorodinsky-Winternitz potential [30]. Here, we make use of the ansatz approach to deal with this complicated equation. A survey on the application of this technique to other wave equations including Dirac, Schrödinger, KleinGordon, spinless-Salpeter, and DKP equations can be found in [31-39]. The paper is organized as follows. In Section 2, we give a brief introduction of the supersymmetry quantum mechanics (SUSYQM). In Section 3, the Dirac equation is written for spin and pseudospin including the Cornell tensor 
interaction term. We next propose a physical ansatz solution to the equation and we solve the Dirac equation under these symmetries in Section 4. Finally, conclusion is presented in Section 5.

\section{Dirac Equation including Tensor Coupling}

In spherical coordinates, the Dirac equation with both scalar potential $S(r)$ and vector potential $V(r)$ can be expressed as $[1,2]$

$$
\begin{aligned}
{[\vec{\alpha} \cdot \vec{p}} & +\beta(M+S(r))-i \beta \vec{\alpha} \cdot \widehat{r} U(r)] \psi(\vec{r}) \\
& =[E-V(r)] \psi(\vec{r})
\end{aligned}
$$

where $E$ is the relativistic energy of the system; $\alpha$ and $\beta$ are the $4 \times 4$ Dirac matrices; $\mathbf{p}$ is the momentum operator, $\vec{p}=-i \vec{\nabla}$. For a particle in a spherical field, the total angular momentum operator $J$ and spin-orbit matrix operator $K=(\vec{\sigma} \cdot \vec{L}+1)$ commute with the Dirac Hamiltonian, where $\sigma$ and $L$ are the Pauli matrix and orbital angular momentum, respectively. The eigenvalues of $K$ are $\kappa=-(j+1 / 2)$ for aligned spin $\left(s_{1 / 2}\right.$, $p_{3 / 2}$, etc. $)$ and $\kappa=(j+1 / 2)$ for unaligned $\operatorname{spin}\left(p_{1 / 2}, d_{3 / 2}\right.$, etc. $)$. The complete set of the conservative quantities can be taken as $\left(H^{2}, K, J^{2}, J_{z}\right)$. As shown in [15], by taking the spherically symmetric Dirac spinor wave functions as

$$
\psi_{n k}(\vec{r})=\left(\begin{array}{l}
f_{n k}(\vec{r}) \\
g_{n k}(\vec{r})
\end{array}\right)=\left(\begin{array}{c}
\frac{F_{n k}(\vec{r})}{r} Y_{j m}^{\ell}(\theta, \varphi) \\
i \frac{G_{n k}(\vec{r})}{r} Y_{j m}^{\tilde{\ell}}(\theta, \varphi)
\end{array}\right),
$$

where $F_{n k}(\vec{r})$ and $G_{n k}(\vec{r})$ are the radial wave functions of the upper and lower components, respectively, $Y_{j m}^{\ell}(\theta, \varphi)$ and $Y_{j m}^{\tilde{\ell}}(\theta, \varphi)$, respectively, stand for spin and pseudospin spherical harmonics that are coupled to the angular momentum $j$ and $m$ is the projection of the angular momentum on the $z$ axis. The orbital angular momentum quantum numbers $\ell$ and $\tilde{\ell}$ refer to the upper and lower components, respectively. The quasidegenerate doublet structure can be expressed in terms of pseudospin angular momentum $s=1 / 2$ and pseudoorbital angular momentum $\tilde{\ell}$, which is defined as $\widetilde{\ell}=\ell+1$ for aligned spin $j=\tilde{\ell}-1 / 2$ and $\tilde{\ell}=\ell-1$ for unaligned spin $j=\tilde{\ell}+1 / 2$. As shown in $[1,2]$, substituting (2) into (1) yields two coupled differential equations as follows:

$$
\begin{aligned}
\left(\frac{d}{d r}\right. & \left.+\frac{\kappa}{r}-U(r)\right) F_{n k}(r) \\
& =\left(M+E_{n k}-V(r)+S(r)\right) G_{n k}(r) \\
\left(\frac{d}{d r}\right. & \left.-\frac{\kappa}{r}+U(r)\right) G_{n k}(r) \\
& =\left(M-E_{n k}+V(r)+S(r)\right) F_{n k}(r)
\end{aligned}
$$

We consider the difference potential $\Delta(r)$ and sum potential $\Sigma(r)$ as $\Delta(r)=V(r)-S(r)$ and $\Sigma(r)=V(r)+S(r)$, respectively.
The spin-orbit quantum number $\kappa$ is related to the orbital angular momentum quantum number $\ell$. By eliminating $G_{n k}(\vec{r})$ in (3) and $F_{n k}(\vec{r})$ in (4), we obtain the following two second-order differential equations for the upper and lower components:

$$
\begin{gathered}
\left\{\frac{d^{2}}{d r^{2}}-\frac{\kappa(\kappa+1)}{r^{2}}+\frac{2 \kappa}{r} U(r)-\frac{d U(r)}{d r}-U^{2}(r)\right. \\
\left.+\frac{d \Delta(r) / d r}{M+E_{n \kappa}-\Delta(r)}\left(\frac{d}{d r}+\frac{\kappa}{r}-U(r)\right)\right\} F_{n \kappa}(r) \\
=\left(M+E_{n \kappa}-\Delta(r)\right)\left(M-E_{n \kappa}+\Sigma(r)\right) F_{n \kappa}(r), \\
\left\{\frac{d^{2}}{d r^{2}}-\frac{\kappa(\kappa-1)}{r^{2}}+\frac{2 \kappa}{r} U(r)+\frac{d U(r)}{d r}-U^{2}(r)\right. \\
\left.+\frac{d \Sigma(r) / d r}{M-E_{n \kappa}+\sum(r)}\left(\frac{d}{d r}-\frac{\kappa}{r}+U(r)\right)\right\} G_{n \kappa}(r) \\
=\left(M+E_{n \kappa}-\Delta(r)\right)\left(M-E_{n \kappa}+\Sigma(r)\right) G_{n \kappa}(r) .
\end{gathered}
$$

We have $\kappa(\kappa-1)=\tilde{\ell}(\tilde{\ell}+1)$ and $\kappa(\kappa+1)=\ell(\ell+1)$.

\section{Pseudospin and Spin Symmetric Solutions}

3.1. Pseudospin Symmetry Limit. The pseudospin symmetry occurs when $d \Sigma(r) / d r=0$ or equivalently $\Sigma(r)=C_{\mathrm{ps}}=$ Const. $[1,2]$. Here, we work on

$$
\Delta(r)=A r^{2}-2 g \frac{2 r^{2}-1}{\left(2 r^{2}+1\right)^{2}}
$$

For the tensor term, we consider the Cornell potential

$$
U(r)=a_{\mathrm{ps}} r+\frac{b_{\mathrm{ps}}}{r} .
$$

Substitution of these two terms into (6) gives

$$
\begin{aligned}
\left\{\frac{d^{2}}{d r^{2}}+\frac{1}{r^{2}}\left(-\kappa(\kappa-1)+2 \kappa b_{\mathrm{ps}}-b_{\mathrm{ps}}-b_{\mathrm{ps}}^{2}\right)\right. \\
\quad-A_{\mathrm{ps}}\left(E_{n \kappa}^{\mathrm{ps}}-M-C_{\mathrm{ps}}\right) r^{2}-a_{\mathrm{ps}}^{2} r^{2} \\
\quad-2 g_{\mathrm{ps}} \frac{2 r^{2}-1}{\left(2 r^{2}+1\right)^{2}}\left(E_{n \kappa}^{\mathrm{ps}}-M-C_{\mathrm{ps}}\right) \\
+\left(E_{n \kappa}^{\mathrm{ps}}+M\right)\left(E_{n \kappa}^{\mathrm{ps}}-M-C_{\mathrm{ps}}\right)-2 \kappa a_{\mathrm{ps}} \\
\left.+a_{\mathrm{ps}}-2 a_{\mathrm{ps}} b_{\mathrm{ps}}\right\} G_{n \kappa}^{\mathrm{ps}}(r)=0,
\end{aligned}
$$

where $\kappa=-\widetilde{\ell}$ and $\kappa=\tilde{\ell}+1$ for $\kappa<0$ and $\kappa>0$, respectively. 
3.2. Spin Symmetry Limit. In the spin symmetry limit $d \Delta(r) /$ $d r=0$ or $\Delta(r)=C_{s}=$ Const. [15], we consider

$$
\begin{gathered}
\sum(r)=A r^{2}-2 g \frac{2 r^{2}-1}{\left(2 r^{2}+1\right)^{2}}, \\
U(r)=a_{s} r+\frac{b_{s}}{r} .
\end{gathered}
$$

Substitution of the later into (5) gives

$$
\begin{aligned}
& \left\{\frac{d^{2}}{d r^{2}}+\frac{1}{r^{2}}\left(-\kappa(\kappa+1)+2 \kappa b_{s}+b_{s}-b_{s}^{2}\right)\right. \\
& -A_{s}\left(M+E_{n \kappa}^{s}-C_{s}\right) r^{2}-a_{s}^{2} r^{2} \\
& +2 g_{s} \frac{2 r^{2}-1}{\left(2 r^{2}+1\right)^{2}}\left(M+E_{n \kappa}^{s}-C_{s}\right) \\
& +\left(E_{n \kappa}^{s}-M\right)\left(M+E_{n \kappa}^{s}-C_{s}\right)+2 \kappa a_{s} \\
& \left.-a_{s}-2 a_{s} b_{s}\right\} F_{n \kappa}^{s}(r)=0,
\end{aligned}
$$

where $\kappa=\ell$ and $\kappa=-\ell-1$ for $\kappa<0$ and $\kappa>0$, respectively.

\section{The Ansatz Solution}

4.1. Solution of the Pseudospin Symmetry Limit. In the previous section, we obtained a Schrodinger-like equation of the form

$$
\begin{aligned}
& \frac{d^{2} G_{n \kappa}^{\mathrm{ps}}(r)}{d r^{2}} \\
& \quad+\left[\varepsilon^{\mathrm{ps}}+\frac{\lambda^{\mathrm{ps}}}{r^{2}}+\chi^{\mathrm{ps}} r^{2}+\delta^{\mathrm{ps}} \frac{2 r^{2}-1}{\left(2 r^{2}+1\right)^{2}}\right] G_{n \kappa}^{\mathrm{ps}}(r)=0,
\end{aligned}
$$

where

$$
\begin{aligned}
& \mathcal{\varepsilon}^{\mathrm{ps}}=\left(E_{n \kappa}^{\mathrm{ps}}+M\right)\left(E_{n \kappa}^{\mathrm{ps}}-M-C_{\mathrm{ps}}\right)-2 \kappa a_{\mathrm{ps}}+a_{\mathrm{ps}}-2 a_{\mathrm{ps}} b_{\mathrm{ps}}, \\
& \lambda^{\mathrm{ps}}=-\kappa(\kappa-1)+2 \kappa b_{\mathrm{ps}}-b_{\mathrm{ps}}-b_{\mathrm{ps}}^{2}, \\
& \chi^{\mathrm{ps}}=-A_{\mathrm{ps}}\left(E_{n \kappa}^{\mathrm{ps}}-M-C_{\mathrm{ps}}\right)-a_{\mathrm{ps}}^{2}, \\
& \delta^{\mathrm{ps}}=-2 g_{\mathrm{ps}}\left(E_{n \kappa}^{\mathrm{ps}}-M-C_{\mathrm{ps}}\right) .
\end{aligned}
$$

The latter fails to admit exact analytical solutions. Therefore, we follow the ansatz approach with the starting square

$$
G_{n \kappa}^{\mathrm{ps}}(r)=f_{n}^{\mathrm{ps}}(r) \exp \left(g_{\kappa}^{\mathrm{ps}}(r)\right),
$$

where

$$
\begin{gathered}
f_{n}^{\mathrm{ps}}(r)= \begin{cases}1, & \text { if } n=0, \\
\prod_{i=1}^{n}\left(r-\alpha_{i}^{n}\right), & \text { if } n \geq 1,\end{cases} \\
g_{\kappa}^{\mathrm{ps}}(r)=-\alpha^{\mathrm{ps}} r^{2}+\beta^{\mathrm{ps}} \ln r+\gamma^{\mathrm{ps}} \ln \left(2 r^{2}+1\right), \quad \alpha^{\mathrm{ps}}>0 .
\end{gathered}
$$

By substitution of $f_{n}(r)$ and $g_{\kappa}(r)$ into (14) and then taking the second-order derivative of the obtained equation, we can get

$$
\begin{aligned}
G_{n \kappa}^{\mathrm{ps} \prime \prime}(r)=\left[g_{\kappa}^{\mathrm{ps} \prime \prime}(r)+g_{\kappa}^{\mathrm{ps} / 2}(r)\right. & \\
& \left.+\frac{f_{n}^{\mathrm{ps} \prime \prime}(r)+2 g_{\kappa}^{\mathrm{ps} \prime}(r) f_{n}^{\mathrm{ps} \prime}(r)}{f_{n}^{\mathrm{ps}}(r)}\right] G_{n \kappa}^{\mathrm{ps}}(r) .
\end{aligned}
$$

By considering the case $n=0$, from (14)-(16), we find

$$
\begin{aligned}
G_{0 \kappa}^{\mathrm{ps} \prime \prime}(r)=\{ & \frac{1}{r^{2}}\left[-\beta^{\mathrm{ps}}+\beta^{\mathrm{ps} 2}\right]+\frac{1}{\left(2 r^{2}+1\right)^{2}} \\
& \times\left[16 \gamma^{\mathrm{ps} 2} r^{2}+16 \alpha^{\mathrm{ps}} \gamma^{\mathrm{ps}} r^{2}+16 \beta^{\mathrm{ps}} \gamma^{\mathrm{ps}} r^{2}\right. \\
& \left.\quad-8 \gamma^{\mathrm{ps}} r^{2}+4 \gamma^{\mathrm{ps}}+8 \alpha^{\mathrm{ps}} \gamma+8 \beta^{\mathrm{ps}} \gamma^{\mathrm{ps}}\right] \\
+ & 4 \alpha^{\mathrm{ps} 2} r^{2}-2 \alpha^{\mathrm{ps}} \\
& \left.-4 \alpha^{\mathrm{ps}} \beta^{\mathrm{ps}}-8 \alpha^{\mathrm{ps}} \gamma^{\mathrm{ps}}\right\} G_{0 \kappa}^{\mathrm{ps}}(r) .
\end{aligned}
$$

By comparing the corresponding powers of (12) and (18), we have

$$
\begin{aligned}
-\lambda^{\mathrm{ps}} & =\beta^{\mathrm{ps}}\left(\beta^{\mathrm{ps}}-1\right), \\
-\chi^{\mathrm{ps}} & =4 \alpha^{\mathrm{ps} 2}, \\
-2 \delta^{\mathrm{ps}} & =16 \gamma^{\mathrm{ps} 2}+16 \alpha^{\mathrm{ps}} \gamma^{\mathrm{ps}}+16 \beta^{\mathrm{ps}} \gamma^{\mathrm{ps}}-8 \gamma^{\mathrm{ps}}, \\
\delta^{\mathrm{ps}} & =4 \gamma^{\mathrm{ps}}+8 \alpha^{\mathrm{ps}} \gamma+8 \beta^{\mathrm{ps}} \gamma^{\mathrm{ps}}, \\
-\varepsilon^{\mathrm{ps}} & =-2 \alpha^{\mathrm{ps}}-4 \alpha^{\mathrm{ps}} \beta^{\mathrm{ps}}-8 \alpha^{\mathrm{ps}} \gamma^{\mathrm{ps}} .
\end{aligned}
$$

Equation (19) gives

$$
\begin{aligned}
\beta^{\mathrm{ps}} & =\frac{1}{2}\left(1+\sqrt{1-4 \lambda^{\mathrm{ps}}}\right) \\
\alpha^{\mathrm{ps}} & =\frac{1}{2} \sqrt{-\chi^{\mathrm{ps}}} \\
\gamma^{\mathrm{ps}} & =-2\left(\alpha^{\mathrm{ps}}+\beta^{\mathrm{ps}}\right) .
\end{aligned}
$$

Actually, to have well-behaved solutions of the radial wave function at boundaries, namely, the origin and the infinity, we need to take $\delta^{\text {ps }}$ from (19) as

$$
\delta^{\mathrm{ps}}=4 \gamma^{\mathrm{ps}}+8 \alpha^{\mathrm{ps}} \gamma+8 \beta^{\mathrm{ps}} \gamma^{\mathrm{ps}}
$$

Form (13), (19), and (20), the ground-state energy satisfies

$$
\begin{aligned}
\varepsilon^{\mathrm{ps}}- & \sqrt{-\chi^{\mathrm{ps}}}-\sqrt{-\chi^{\mathrm{ps}}}\left(1+\sqrt{1-4 \lambda^{\mathrm{ps}}}\right) \\
& +4 \sqrt{-\chi^{\mathrm{ps}}}\left(\sqrt{-\chi^{\mathrm{ps}}}+\left(1+\sqrt{1-4 \lambda^{\mathrm{ps}}}\right)\right)=0 .
\end{aligned}
$$


TABLE 1: Bound state for the pseudospin symmetry $a_{\mathrm{ps}}=-0.02, b_{\mathrm{ps}}=0.01, M=0.5 \mathrm{fm}^{-1}$, and $C_{\mathrm{ps}}=-1 \mathrm{fm}^{-1}$.

\begin{tabular}{llcrr}
\hline$\tilde{\ell}$ & $\kappa$ & $(\ell, j)$ & $A=-0.05$ & $E_{0 \kappa}^{\mathrm{ps}}\left(\mathrm{fm}^{-1}\right)$ \\
\hline 1 & -1 & $0 S_{1 / 2}$ & -0.492577461 & -0.499257852 \\
2 & -2 & $0 P_{3 / 2}$ & -0.492679105 & -0.499267983 \\
3 & -3 & $0 d_{5 / 2}$ & -0.492730875 & -0.499273142 \\
4 & -4 & $0 f_{7 / 2}$ & -0.492762185 & -0.499276263 \\
\hline
\end{tabular}

TABLE 2: Bound state for the spin symmetry $a_{s}=0.02, b_{s}=0.01, M=0.5 \mathrm{fm}^{-1}$, and $C_{s}=1 \mathrm{fm}^{-1}$.

\begin{tabular}{lcccr}
\hline$\ell$ & $\kappa$ & $(\ell, j)$ & $A=-0.05$ & $E_{0 \kappa}^{s}\left(\mathrm{fm}^{-1}\right)$ \\
\hline 1 & & $0 p_{3 / 2}$ & 0.506363937 & 0.500636263 \\
2 & -2 & $0 d_{5 / 2}$ & 0.506651467 & 0.500665063 \\
3 & -3 & $0 f_{7 / 2}$ & 0.506779923 & 0.500677931 \\
4 & -4 & $0 g_{9 / 2}$ & 0.50685245 & 0.500685197 \\
\hline
\end{tabular}

TABLE 3: Energies in the pseudospin symmetry limit for $A_{\mathrm{ps}}=-0.5, a_{\mathrm{ps}}=-0.02, b_{\mathrm{ps}}=0.01$, and $M=0.5 \mathrm{fm}^{-1}$.

\begin{tabular}{lcccc}
\hline$C_{\mathrm{ps}}$ & & \multicolumn{3}{c}{$E_{0 \kappa}^{\mathrm{ps}}\left(\mathrm{fm}^{-1}\right)$} \\
$0 S_{1 / 2}$ & $0 P_{3 / 2}$ & $0 d_{5 / 2}$ & -4.49927873 \\
\hline-5 & -4.499263699 & -4.49927201 & -4.499276204 & -3.499278111 \\
-4 & -3.499262219 & -3.499270997 & -3.499275435 & -2.499277494 \\
-3 & -2.499260752 & -2.499269988 & -2.499274669 & -1.499276877 \\
-2 & -1.499259296 & -1.499268983 & -1.499273904 & -0.499276263 \\
-1 & -0.499257852 & -0.499267983 & -0.499273142 & 0.500724351 \\
0 & 0.50074358 & 0.500733013 & 0.500727617 & \\
\hline
\end{tabular}

From (14), (16), and (20), we simply have the upper and lower components of the wave function as

$$
\begin{aligned}
& g_{\kappa}^{\mathrm{ps}}(r)=-\frac{1}{2} \sqrt{-\chi^{\mathrm{ps}}} r^{2}+\frac{1}{2}\left(1+\sqrt{1-4 \lambda^{\mathrm{ps}}}\right) \\
& \times \ln r-2\left(\alpha^{\mathrm{ps}}+\beta^{\mathrm{ps}}\right) \ln \left(2 r^{2}+1\right), \\
& G_{0 \kappa}^{\mathrm{ps}}(r)= N_{0 \kappa} r^{(1 / 2)\left(1+\sqrt{1-4 \lambda^{\mathrm{ps}}}\right)}\left(2 r^{2}+1\right)^{-2\left(\alpha^{\mathrm{ps}}+\beta^{\mathrm{ps}}\right)} \\
& \times \exp \left(-\frac{1}{2} \sqrt{-\chi^{\mathrm{ps}}} r^{2}\right), \\
& F_{0 \kappa}^{\mathrm{ps}}(r)=\frac{1}{M-} E_{0 \kappa}^{\mathrm{ps}}+C_{\mathrm{ps}}\left(\frac{d}{d r}-\frac{\kappa}{r}+U(r)\right) G_{0 \kappa}^{\mathrm{ps}}(r) .
\end{aligned}
$$

4.2. Solution of the Spin Symmetry Limit. In this case, our ordinary differential equation is

$$
\frac{d^{2} F_{n \kappa}^{s}(r)}{d r^{2}}+\left[\varepsilon^{s}+\frac{\lambda^{s}}{r^{2}}+\chi^{s} r^{2}+\delta^{s} \frac{2 r^{2}-1}{\left(2 r^{2}+1\right)^{2}}\right] F_{n \kappa}^{s}(r)=0
$$

where

$$
\begin{aligned}
& \varepsilon^{s}=\left(E_{n \kappa}^{s}-M\right)\left(M+E_{n \kappa}^{s}-C_{s}\right)+2 \kappa a_{s}-a_{s}-2 a_{s} b_{s}, \\
& \lambda^{s}=-\kappa(\kappa+1)+2 \kappa b_{s}+b_{s}-b_{s}^{2}, \\
& \chi^{s}=-A_{s}\left(M+E_{n \kappa}^{s}-C_{s}\right)-a_{s}^{2}, \\
& \delta^{s}=2 g_{s}\left(M+E_{n \kappa}^{s}-C_{s}\right) .
\end{aligned}
$$

In this case, we introduce the ansatz

$$
F_{n \kappa}^{\mathcal{S}}(r)=f_{n}^{\mathcal{s}}(r) \exp \left(g_{\kappa}^{s}(r)\right)
$$

where

$$
\begin{gathered}
f_{n}^{s}(r)= \begin{cases}1, & \text { if } n=0, \\
\prod_{i=1}^{n}\left(r-\alpha_{i}^{n}\right), & \text { if } n \geq 1,\end{cases} \\
g_{\kappa}^{s}(r)=-\alpha^{s} r^{2}+\beta^{s} \ln r+\gamma^{s} \ln \left(2 r^{2}+1\right), \quad \alpha^{s}>0,
\end{gathered}
$$

substitution of the proposed ansatz gives

$$
\begin{aligned}
& F_{n \kappa}^{s \prime \prime}(r) \\
& =\left[g_{\kappa}^{s^{\prime \prime}}(r)+g_{\kappa}^{s^{\prime 2}}(r)+\frac{f_{n}^{s^{\prime \prime}}(r)+2 g_{\kappa}^{s^{\prime}}(r) f_{n}^{s^{\prime}}(r)}{f_{n}^{s}(r)}\right] F_{n \kappa}^{s}(r) .
\end{aligned}
$$


TABLE 4: Energies in the spin symmetry limit for $A_{s}=-0.5, a_{s}=0.02, b_{s}=0.01$, and $M=0.5 \mathrm{fm}^{-1}$.

\begin{tabular}{|c|c|c|c|c|}
\hline \multirow{2}{*}{$C_{s}$} & \multicolumn{4}{|c|}{$E_{0 \kappa}^{s}\left(\mathrm{fm}^{-1}\right)$} \\
\hline & $0 p_{3 / 2}$ & $0 d_{5 / 2}$ & $0 f_{7 / 2}$ & $0 g_{9 / 2}$ \\
\hline 0 & -0.499365805 & -0.499336214 & -0.499322986 & -0.499315517 \\
\hline 1 & 0.500636263 & 0.500665063 & 0.500677931 & 0.500685197 \\
\hline 2 & 1.500638332 & 1.500666338 & 1.500678847 & 1.500685909 \\
\hline 3 & 2.500640401 & 2.500667612 & 2.500679761 & 2.500686621 \\
\hline 4 & 3.50064247 & 3.500668885 & 3.500680675 & 3.500687332 \\
\hline 5 & 4.500644539 & 4.500670157 & 4.500681588 & 4.500688043 \\
\hline
\end{tabular}

TABLE 5: Energies in the pseudospin symmetry limit for $A_{\mathrm{ps}}=-0.5, a_{\mathrm{ps}}=-0.02, b_{\mathrm{ps}}=0.01$, and $C_{\mathrm{ps}}=-1 \mathrm{fm}^{-1}$.

\begin{tabular}{|c|c|c|c|c|}
\hline \multirow{2}{*}{$M\left(\mathrm{fm}^{-1}\right)$} & \multicolumn{4}{|c|}{$E_{0 \kappa}^{\mathrm{ps}}\left(\mathrm{fm}^{-1}\right)$} \\
\hline & $0 S_{1 / 2}$ & $0 P_{3 / 2}$ & $0 d_{5 / 2}$ & $0 f_{7 / 2}$ \\
\hline 0 & -0.999259296 & -0.999268983 & -0.999273904 & -0.999276877 \\
\hline 0.2 & -0.799258717 & -0.799268582 & -0.799273599 & -0.799276631 \\
\hline 0.4 & -0.59925814 & -0.599268183 & -0.599273295 & -0.599276385 \\
\hline 0.6 & -0.399257564 & -0.399267783 & -0.39927299 & -0.39927614 \\
\hline 0.8 & -0.199256991 & -0.199267385 & -0.199272686 & -0.199275894 \\
\hline
\end{tabular}

TABLE 6: Energies in the spin symmetry limit for $A_{s}=-0.5, a_{s}=0.02, b_{s}=0.01$, and $C_{s}=1 \mathrm{fm}^{-1}$.

\begin{tabular}{|c|c|c|c|c|}
\hline \multirow{2}{*}{$M\left(\mathrm{fm}^{-1}\right)$} & \multicolumn{4}{|c|}{$E_{n \kappa}^{s^{+}}\left(\mathrm{fm}^{-1}\right)$} \\
\hline & $0 p_{3 / 2}$ & $0 d_{5 / 2}$ & $0 f_{7 / 2}$ & $0 g_{9 / 2}$ \\
\hline 0 & 1.000638332 & 1.000666338 & 1.000678847 & 1.000685909 \\
\hline 0.2 & 0.800637505 & 0.800665828 & 0.80067848 & 0.800685624 \\
\hline 0.4 & 0.600636677 & 0.600665318 & 0.600678114 & 0.600685339 \\
\hline 0.6 & 0.40063585 & 0.400664807 & 0.400677748 & 0.400685054 \\
\hline 0.8 & 0.200635022 & 0.200664297 & 0.200677381 & 0.200684769 \\
\hline
\end{tabular}

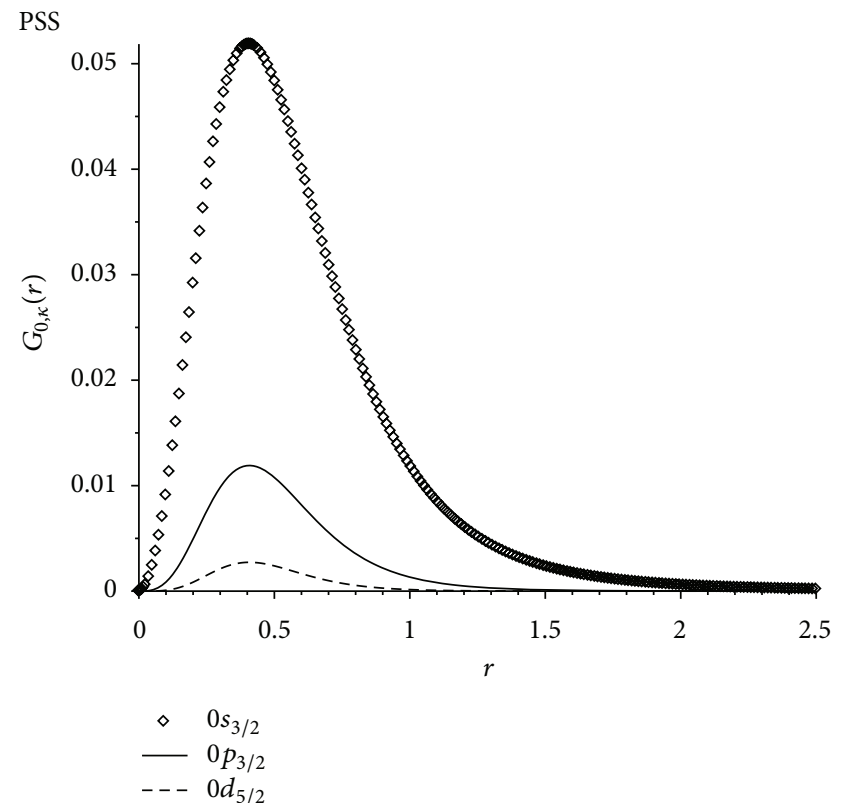

(a)

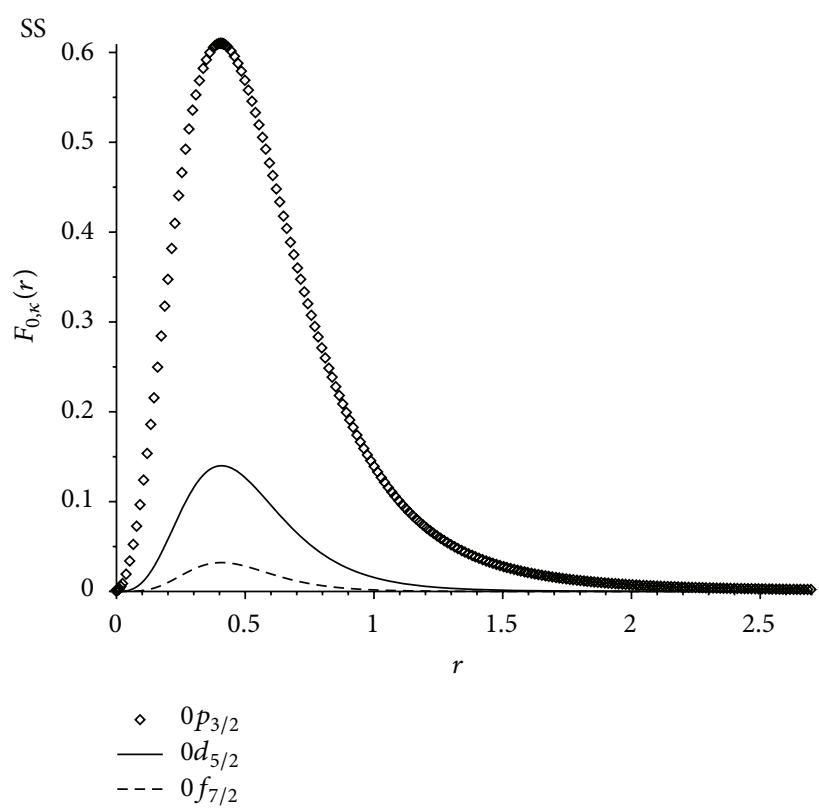

(b)

FIGURE 1: PSS: wavefunction for pseudospin symmetry limit for $A_{\mathrm{ps}}=-0.5, a_{\mathrm{ps}}=-0.02, b_{\mathrm{ps}}=0.01, M=0.5 \mathrm{fm}^{-1}$, and $C_{\mathrm{ps}}=-1 \mathrm{fm}{ }^{-1}$. SS: wavefunction for spin symmetry limit for $A_{s}=-0.5, a_{s}=0.02, b_{s}=0.01, M=0.5 \mathrm{fm}^{-1}$, and $C_{s}=1 \mathrm{fm}^{-1}$. 
For $n=0$, we have

$$
\begin{aligned}
& F_{0 \kappa}^{s \prime \prime}(r) \\
& =\left\{\frac{1}{r^{2}}\left[-\beta^{s}+\beta^{s^{2}}\right]+\frac{1}{\left(2 r^{2}+1\right)^{2}}\right. \\
& \times\left[16 \gamma^{s^{2}} r^{2}+16 \alpha^{s} \gamma^{s} r^{2}+16 \beta^{s} \gamma^{s} r^{2}\right. \\
& \left.-8 \gamma^{s} r^{2}+4 \gamma^{s}+8 \alpha^{s} \gamma^{s}+8 \beta^{s} \gamma^{s}\right] \\
& \left.+4 \alpha^{s 2} r^{2}-2 \alpha^{s}-4 \alpha^{s} \beta^{s}-8 \alpha^{s} \gamma^{s}\right\} F_{0 \kappa}^{s}(r) \text {. }
\end{aligned}
$$

By comparing the corresponding powers of (24) and (30), we have

$$
\begin{aligned}
& \beta^{s}=\frac{1}{2}\left(1+\sqrt{1-4 \lambda^{s}}\right), \\
& \alpha^{s}=\frac{1}{2} \sqrt{-\chi^{s}}, \\
& \gamma^{s}=-2\left(\alpha^{s}+\beta^{s}\right), \\
& \varepsilon^{s}-2 \alpha^{s}-4 \alpha^{s} \beta^{s}-8 \alpha^{s} \gamma^{s}=0 .
\end{aligned}
$$

To have physically acceptable solutions, we pick up the value by considering the above equation; the first node eigenvalue satisfies

$$
\begin{aligned}
\mathcal{E}^{s} & -\sqrt{-\chi^{s}}-\sqrt{-\chi^{s}}\left(1+\sqrt{1-4 \lambda^{s}}\right) \\
& +4 \sqrt{-\chi^{s}}\left(\sqrt{-\chi^{s}}+\left(1+\sqrt{1-4 \lambda^{s}}\right)\right)=0 .
\end{aligned}
$$

From (26), (28), and (31), the upper component of the wave function is

$$
\begin{aligned}
g_{\kappa}^{s}(r)= & -\frac{1}{2} \sqrt{-\chi^{s}} r^{2} \\
& +\frac{1}{2}\left(1+\sqrt{1-4 \lambda^{s}}\right) \ln r-2\left(\alpha^{s}+\beta^{s}\right) \ln \left(2 r^{2}+1\right) \\
F_{0 \kappa}^{s}(r)= & N_{0 \kappa} r^{(1 / 2)\left(1+\sqrt{1-4 \lambda^{s}}\right)} \\
& \times\left(2 r^{2}+1\right)^{-2\left(\alpha^{s}+\beta^{s}\right)} \exp \left(-\frac{1}{2} \sqrt{-\chi^{s} r^{2}}\right)
\end{aligned}
$$

and for the lower component of the wave function, we have

$$
G_{0 \kappa}^{s}(r)=\frac{1}{M+E_{0 \kappa}^{s}-C_{s}}\left(\frac{d}{d r}+\frac{\kappa}{r}-U(r)\right) F_{0 \kappa}^{s}(r) .
$$

We have given some numerical values of the energy eigenvalues in Tables 1-6 for various states. We have investigated the dependence of the bound-state energy levels $n=0$ on potential parameter $A$. The results in Tables 1 and 2 have found that case $A=-0.05$ is contrary to case $A=-0.5$ under the condition of the pseudospin and spin symmetries, respectively. Tables 3 and 4 present the dependence of the bound-state energy levels on parameters $C_{\mathrm{ps}}$ and $C_{s}$ in view of the pseudospin and spin symmetry limits. It is seen in Tables 3 and 4 that although bound states obtained in view of spin symmetry become more bounded with increasing $C_{s}$, they become less bounded in the pseudospin symmetry limit with increasing $C_{\mathrm{ps}}$. We show the effects of the $M$-parameter on the bound states under the conditions of the pseudospin and spin symmetry limits. The results are given in Tables 5 and 6 . It is seen that if the $M$-parameter increases, the bound states become less bounded for both the pseudospin and the spin symmetry limits. In Figure 1, the wave functions are plotted for spin and pseudospin symmetry limits.

\section{Conclusion}

In this paper, we have obtained the approximate solutions of the Dirac equation for the isotonic oscillator potential including the Cornell tensor interaction within the framework of pseudospin and spin symmetry limits using the ansatz approach which stands as a strong tool of mathematical physics. We have obtained the energy eigenvalues and corresponding lower and upper wave functions.

\section{Conflict of Interests}

The authors declare that there is no conflict of interests regarding the publication of this paper.

\section{Acknowledgment}

The authors wish to give their sincere gratitude to the referees for a technical comment on the paper.

\section{References}

[1] J. N. Ginocchio, "Relativistic symmetries in nuclei and hadrons," Physics Reports, vol. 414, no. 4-5, pp. 165-261, 2005.

[2] D. Troltenier, C. Bahri, and J. P. Draayer, "Generalized pseudoSU(3) model and pairing," Nuclear Physics A, vol. 586, no. 1, pp. 53-72, 1995.

[3] J. N. Ginocchio, "Pseudospin as a relativistic symmetry," Physical Review Letters, vol. 78, no. 3, pp. 436-439, 1997.

[4] J. N. Ginocchio, A. Leviatan, J. Meng, and S.-G. Zhou, "Test of pseudospin symmetry in deformed nuclei," Physical Review C, vol. 69, no. 3, Article ID 034303, 2004.

[5] S.-H. Dong and M. Lozada-Cassou, "Generalized hypervirial and recurrence relations for radial matrix elements in arbitrary dimensions," Modern Physics Letters A: Particles and Fields, Gravitation, Cosmology, Nuclear Physics, vol. 20, no. 20, pp. 1533-1540, 2005.

[6] G.-F. Wei, C.-Y. Long, and S.-H. Dong, "The scattering of the Manning-Rosen potential with centrifugal term," Physics Letters A, vol. 372, no. 15, pp. 2592-2596, 2008.

[7] W. C. Qiang and S. H. Dong, "The Manning-Rosen potential studied by a new approximate scheme to the centrifugal term," Physica Scripta, vol. 79, Article ID 045004, 2009.

[8] A. F. Nikiforov and V. B. Uvarov, Special Functions of Mathematical Physics, Birkhäuser, Basel, Switzerland, 1988. 
[9] G. F. Wei and S. H. Dong, "Algebraic approach to energy spectra of the Scarf type and generalized Pöschl-Teller potentials," Canadian Journal of Physics, vol. 89, no. 12, pp. 1225-1231, 2011.

[10] G.-F. Wei and S.-H. Dong, "Approximately analytical solutions of the Manning-Rosen potential with the spin-orbit coupling term and spin symmetry," Physics Letters A, vol. 373, no. 1, pp. 49-53, 2008.

[11] G. F. Wei and S. H. Dong, "Pseudospin symmetry in the relativistic Manning-Rosen potential including a Pekeris-type approximation to the pseudo-centrifugal term," Physics Letters $B$, vol. 686, no. 4-5, pp. 288-292, 2010.

[12] J. Meng, K. Sugawara-Tanabe, S. Yamaji, and A. Arima, "Pseudospin symmetry in $\mathrm{Zr}$ and $\mathrm{Sn}$ isotopes from the proton drip line to the neutron drip line," Physical Review C, vol. 59, p. 154, 1999.

[13] F. S. Stephen, M. A. Deleplanque, J. E. Draper et al., "Pseudospin symmetry and quantized alignment in nuclei," Physical Review Letters, vol. 65, no. 3, pp. 301-304, 1990.

[14] A. E. Stuchbery, "Magnetic properties of rotational states in the pseudo-Nilsson model," Nuclear Physics A, vol. 700, no. 1-2, pp. 83-116, 2002.

[15] E. Maghsoodi, H. Hassanabadi, S. Zarrinkamar, and H. Rahimov, "Relativistic symmetries of the Dirac equation under the nuclear WoodsSaxon potential," Physica Scripta, vol. 85, no. 5, Article ID 055007, 2012.

[16] C.-S. Jia, P. Guo, and X.-L. Peng, "Exact solution of the DiracEckart problem with spin and pseudospin symmetry," Journal of Physics A: Mathematical and General, vol. 39, no. 24, pp. 77377744, 2006.

[17] C.-S. Jia and A. de Souza Dutra, "Position-dependent effective mass Dirac equations with $P T$-symmetric and non- $P T$ symmetric potentials," Journal of Physics A: Mathematical and General, vol. 39, no. 38, pp. 11877-11887, 2006.

[18] H. Hassanabadi, E. Maghsoodi, S. Zarrinkamar, and H. Rahimov, "Dirac equation for generalized Pöschl-Teller scalar and vector potentials and a Coulomb tensor interaction by Nikiforov-Uvarov method," Journal of Mathematical Physics, vol. 53, no. 2, Article ID 022104, 2012.

[19] H. Akcay, "Dirac equation with scalar and vector quadratic potentials and Coulomb-like tensor potential," Physics Letters: A, vol. 373, no. 6, pp. 616-620, 2009.

[20] C.-S. Jia, T. Chen, and L.-G. Cui, "Approximate analytical solutions of the Dirac equation with the generalized PöschlTeller potential including the pseudo-centrifugal term," Physics Letters A, vol. 373, no. 18-19, pp. 1621-1626, 2009.

[21] L. H. Zhang, X. P. Li, and C. S. Jia, "Approximate analytical solutions of the Dirac equation with the generalized Morse potential model in the presence of the spin symmetry and pseudo-spin symmetry," Physica Scripta, vol. 80, no. 3, Article ID 035003, 2009.

[22] H. Akcay, "The Dirac oscillator with a Coulomb-like tensor potential," Journal of Physics A: Mathematical and Theoretical, vol. 40, no. 24, pp. 6427-6432, 2007.

[23] O. Aydogdu and R. Sever, "Exact pseudospin symmetric solution of the dirac equation for pseudoharmonic potential in the presence of tensor potential," Few-Body Systems, vol. 47, pp. 193200, 2010.

[24] S.-H. Dong, Factorization Method in Quantum Mechanics, Springer, Berlin, Germany, 2007.

[25] J. Meng, K. Sugawara-Tanabe, S. Yamaji, P. Ring, and A. Arima, "Pseudospin symmetry in relativistic mean field theory," Physical Review C, vol. 58, no. 2, article R628, 1998.
[26] K. Thirulogasanther and N. Saad, "Coherent states associated with the wavefunctions and the spectrum of the isotonic oscillator," Journal of Physics A: Mathematical and General, vol. 37, no. 16, p. 4567, 2004.

[27] J.-S. Wang, T.-K. Liu, and M.-S. Zhan, "Nonclassical properties of even and odd generalized coherent states for an isotonic oscillator," Journal of Optics B: Quantum and Semiclassical Optics, vol. 2, no. 6, pp. 758-763, 2000.

[28] O. A. Chalykh and A. P. Veselov, "A remark on rational isochronous potentials," Journal of Nonlinear Mathematical Physics, vol. 12, no. 1, pp. 179-183, 2005.

[29] J. Casahorran, "On a novel supersymmetric connections between harmonic and isotonic oscillators," Physica A, vol. 217, no. 3-4, pp. 429-439, 1995.

[30] C. Grosche, G. S. Pogosyan, and A. N. Sissakian, "Path integral discussion for Smorodinsky-Winternitz potentials: II. The twoand three-dimensional sphere," Fortschritte der Physik/Progress of Physics, vol. 43, no. 6, pp. 523-563, 1995.

[31] N. Akpan, E. Ikot, S. Maghsoodi, H. Zarrinkamar, and Z. Hassanabadi, "Shape-invariant approach to study relativistic symmetries of the dirac equation with a new hyperbolical potential combination," Zeitschrift für Naturforschung A, vol. 68, pp. 499-509, 2013.

[32] H. Hassanabadi, E. Maghsoodi, N. Salehi, A. N. Ikot, and S. Zarrinkamar, "Scattering states of the dirac equation under asymmetric Hulthén potential," The European Physical Journal Plus, vol. 128, no. 10, pp. 1-9, 2013.

[33] S.-H. Dong, "Exact solutions of the two-dimensional Schrdinger equation with certain central potentials," International Journal of Theoretical Physics, vol. 39, no. 4, pp. 1119-1128, 2000.

[34] S. H. Dong, "Correlations of spin states for icosahedral double group," International Journal of Theoretical Physics, vol. 40, no. 2, pp. 569-581, 2001.

[35] H. Hassanabadi and A. A. Rajabi, "Energy levels of a spherical quantum dot in a confining potential," Physics Letters A, vol. 373, no. 6, pp. 679-681, 2009.

[36] H. Hassanabadi and A. A. Rajabi, "Relativistic versus nonrelativistic solution of the $N$-fermion problem in a hyperradiusconfining potential," Few-Body Systems, vol. 41, no. 3-4, pp. 201210, 2007.

[37] S.-H. Dong, Z.-Q. Ma, and G. Esposito, "Exact solutions of the Schrödinger equation with inverse-power potential," Foundations of Physics Letters, vol. 12, no. 5, pp. 465-474, 1999.

[38] H. Hassanabadi, H. Rahimov, and S. Zarrinkamar, "Cornell and Coulomb interactions for the D-dimensional Klein-Gordon equation," Annalen der Physik, vol. 523, no. 7, pp. 566-575, 2011.

[39] D. Agboola and Y.-Z. Zhang, "Unified derivation of exact solutions for a class of quasi-exactly solvable models," Journal of Mathematical Physics, vol. 53, no. 4, Article ID 042101, 13 pages, 2012. 

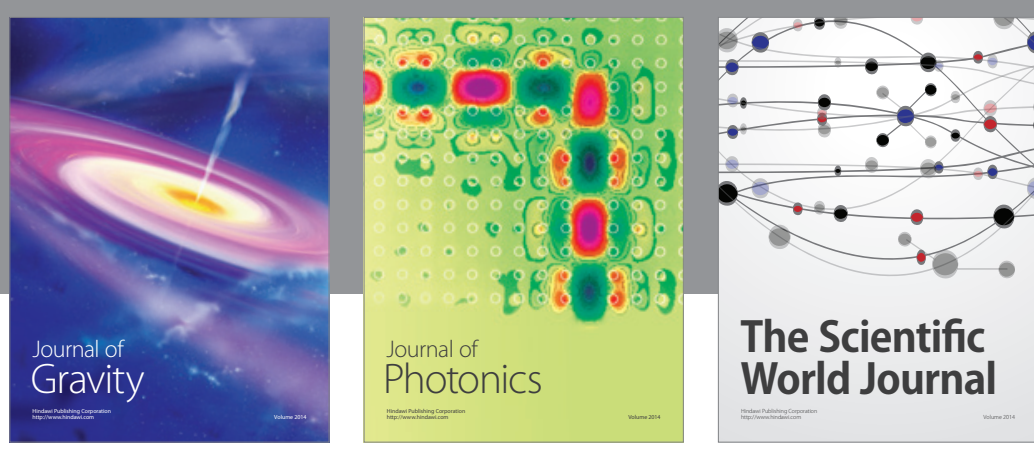

The Scientific World Journal
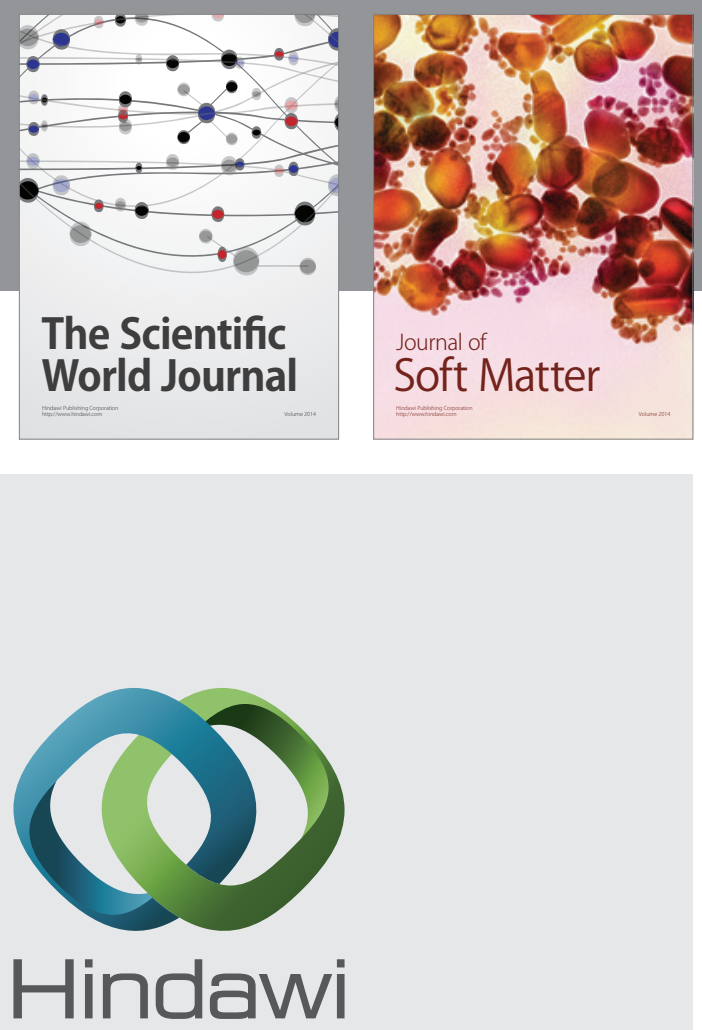

Submit your manuscripts at

http://www.hindawi.com

nternational Journal of

Statistical Mechanics
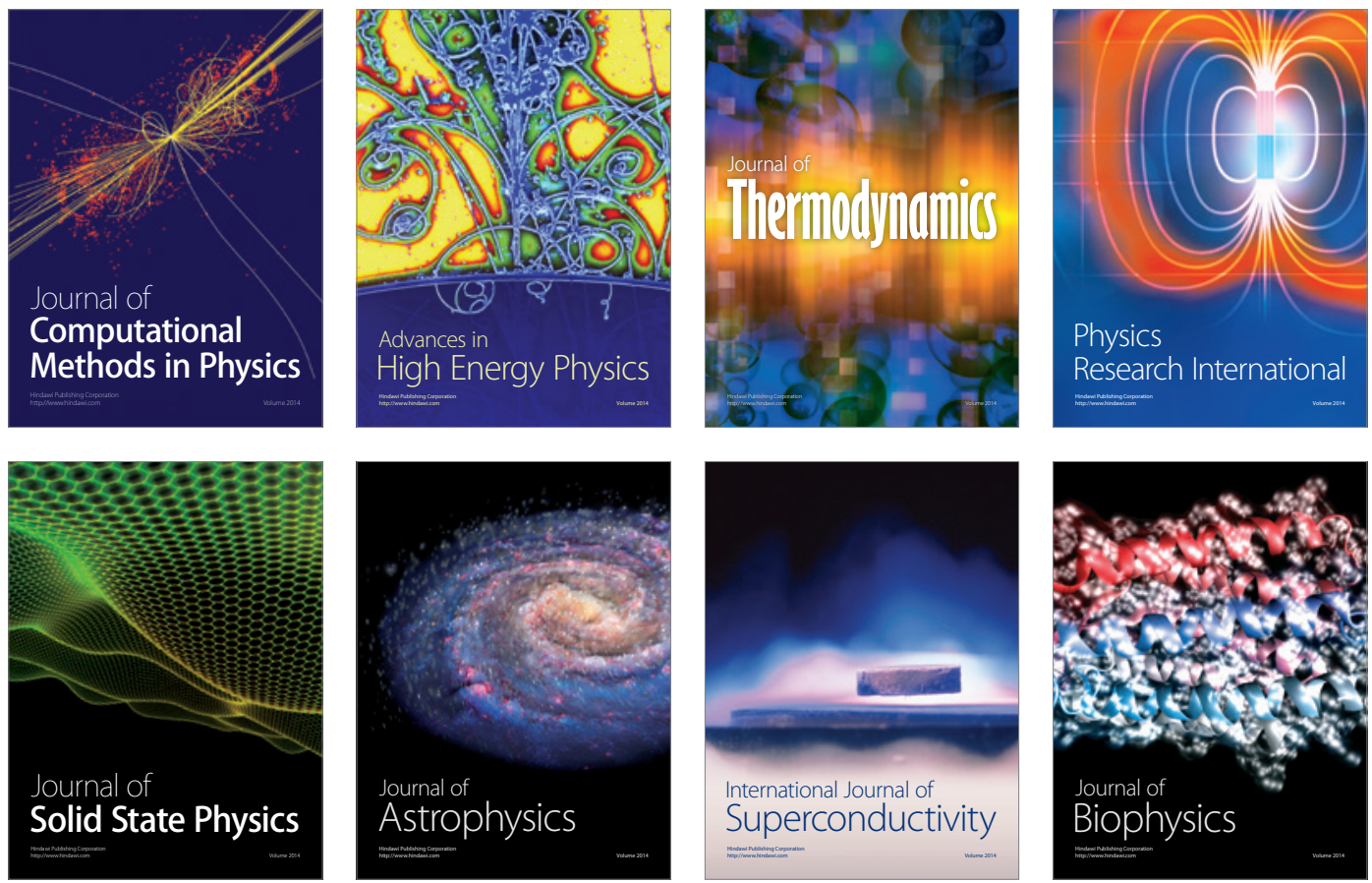
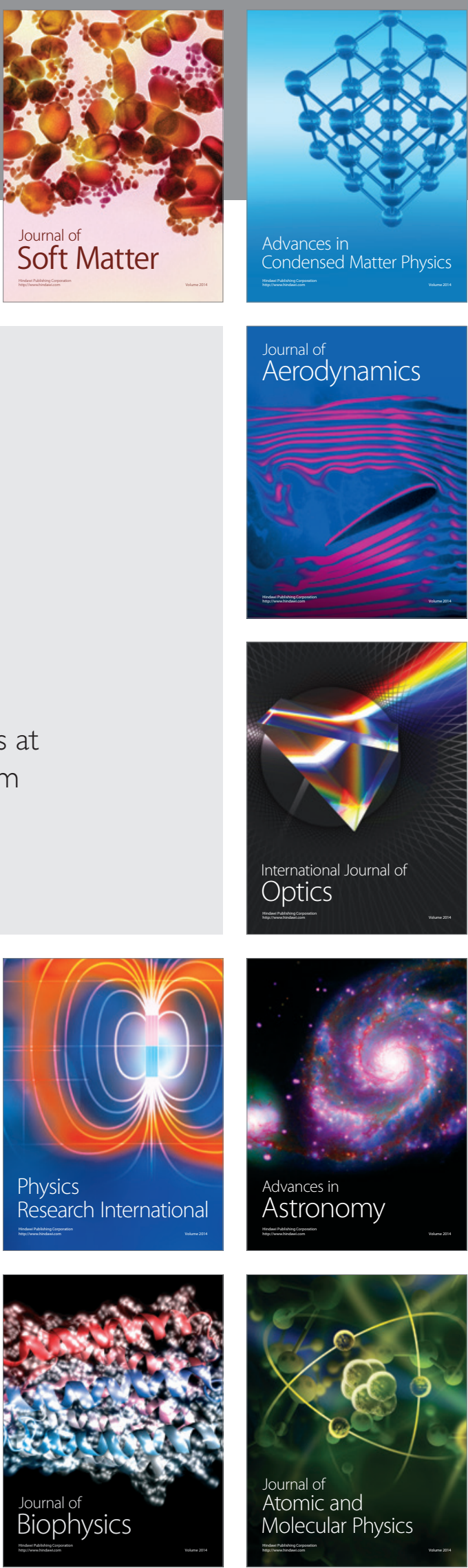